Brandon Michael Henry*, Stefanie W. Benoit, Jens Vikse, Brandon A. Berger, Christina Pulvino, Jonathan Hoehn, James Rose, Maria Helena Santos de Oliveira, Giuseppe Lippi and Justin L. Benoit

\title{
The anti-inflammatory cytokine response characterized by elevated interleukin-10 is a stronger predictor of severe disease and poor outcomes than the pro-inflammatory cytokine response in coronavirus disease 2019 (COVID-19)
}

https://doi.org/10.1515/cclm-2020-1284

Received August 20, 2020; accepted November 12, 2020;

published online November 26, 2020

\section{Abstract}

Objectives: Severe coronavirus disease 2019 (COVID-19) is associated with a dysregulated immune state. While research has focused on the hyperinflammation, little research has been performed on the compensatory antiinflammatory response. The aim of this study was to evaluate the anti-inflammatory cytokine response to COVID-19, by assessing interleukin-10 (IL-10) and IL-10/ lymphocyte count ratio and their association with outcomes.

Methods: Adult patients presenting to the emergency department (ED) with laboratory-confirmed COVID-19 were

\footnotetext{
*Corresponding author: Brandon Michael Henry, MD, Cardiac Intensive Care Unit, The Heart Institute, Cincinnati Children's Hospital Medical Center, 3333 Burnet Ave. 45229, Cincinnati, OH, USA, Phone/Fax: +1 716598 8610, E-mail: brandon.henry@cchmc.org Stefanie W. Benoit, Division of Nephrology and Hypertension, Cincinnati Children's Hospital Medical Center, Cincinnati, OH, USA; Department of Pediatrics, College of Medicine, University of Cincinnati, Cincinnati, OH, USA

Jens Vikse, Clinical Immunology Unit, Stavanger University Hospital, Stavanger, Norway

Brandon A. Berger, Christina Pulvino, Jonathan Hoehn and Justin L. Benoit, Department of Emergency Medicine, University of Cincinnati, Cincinnati, $\mathrm{OH}$, USA

James Rose, Division of Nephrology and Hypertension, Cincinnati Children's Hospital Medical Center, Cincinnati, OH, USA

Maria Helena Santos de Oliveira, Department of Statistics, Federal University of Parana, Curitiba, Brazil

Giuseppe Lippi, Department of Neuroscience, Biomedicine and Movement, Section of Clinical Biochemistry, University of Verona, Verona, Italy. https://orcid.org/0000-0001-9523-9054
}

recruited. The primary endpoint was maximum COVID-19 severity within 30 days of index ED visit.

Results: A total of 52 COVID-19 patients were enrolled. IL-10 and IL-10/lymphocyte count were significantly higher in patients with severe disease $(\mathrm{p}<0.05)$, as well as in those who developed severe acute kidney injury (AKI) and new positive bacterial cultures (all $\mathrm{p} \leq 0.01$ ). In multivariable analysis, a one-unit increase in IL-10 and IL-10/ lymphocyte count were associated with $42 \%(\mathrm{p}=0.031)$ and $32 \%(\mathrm{p}=0.013)$ increased odds, respectively, of severe COVID-19. When standardized to a one-unit standard deviations scale, an increase in the IL-10 was a stronger predictor of maximum 30-day severity and severe AKI than increases in IL-6 or IL-8.

Conclusions: The hyperinflammatory response to COVID-19 is accompanied by a simultaneous anti-inflammatory response, which is associated with poor outcomes and may increase the risk of new positive bacterial cultures. IL-10 and IL-10/lymphocyte count at ED presentation were independent predictors of COVID-19 severity. Moreover, elevated IL-10 was more strongly associated with outcomes than pro-inflammatory IL-6 or IL-8. The anti-inflammatory response in COVID-19 requires further investigation to enable more precise immunomodulatory therapy against SARS-CoV-2.

Keywords: anti-inflammatory response; cytokines; immunoparalysis; outcomes; SARS-CoV-2.

\section{Introduction}

Coronavirus disease 2019 (COVID-19) is caused by the severe acute respiratory syndrome coronavirus-2 (SARS-CoV-2). Early reports of severe COVID-19 cases described a constellation of hyperinflammation and hypercoagulation, culminating in life-threatening immune-mediated and thrombotic 
organ damage. Excessive production of pro-inflammatory cytokines (frequently referred to as "cytokine storm") is considered a central part of the immunopathology in COVID-19 [1], and major efforts have been made to decipher the hyperinflammatory immunophenotype and employ efficient and targeted immunosuppressive treatment strategies. Many authors have argued for the use of tocilizumab, a monoclonal anti-interleukin (IL)-6 receptor antibody, in severe COVID-19 [2]. However, in the recent randomized, double-blind, placebo-controlled phase III COVACTA trial, this drug failed to reach its primary and key secondary endpoints of improved clinical status and reduced mortality, respectively [3]. Moreover, recent studies have raised substantial doubts with respect to the role of hyperinflammation in development of COVID-19 critical illness $[4,5]$. This evidence is hence reflective of a much more complex immunopathology.

Hyperinflammation may often coexist with a compensatory anti-inflammatory response syndrome (CARS) in sepsis and critical illness, which induces quantitative and qualitative defects in the innate and adaptive immune system $[6,7]$. When severe and persistent, CARS is referred to as "immunoparalysis", and has been associated with impaired anti-microbial defense, risk of superinfections and increased mortality $[8,9]$. The immunophenotype of CARS includes increased production of anti-inflammatory cytokines such as IL-10, inefficient antigen presentation, lymphocyte apoptosis and upregulation of inhibitory molecules such as programmed cell death protein (PD)-1 [6]. Molecular analyses in CARS typically reveal downregulation of Human Leukocyte Antigen - DR isotype (HLA-DR) on monocytes, as well as impaired lipopolysaccharide (LPS)-induced tumor necrosis factor (TNF)- $\alpha$ release ex vivo [6].

The relative presence of hyperinflammation and immunoparalysis in COVID-19 is likely to vary among patients and during an individual's course of disease. Safe and efficient immunomodulatory therapy must be tailored to the underlying immunophenotype for preventing iatrogenic deterioration of immune function. Notably, other abnormalities may exacerbate such a functional immunoparalysis in COVID-19. A kaleidoscope of profound blood cells abnormalities have been evidenced in patients with COVID-19, encompassing especially altered myelopoiesis with generation of immature and dysfunctional leukocytes, which may hence be ineffective to counteract secondary bacterial infections [10]. Efforts should hence be made to better characterize the immunobiology of COVID-19 and develop strategies that allow for immunophenotypic stratification of patients.

In this study, we aim to characterize the antiinflammatory cytokine profile, with emphasis on IL-10 and the IL-10/lymphocyte count ratio, in adult patients with laboratory confirmed COVID-19, and associate evidence of CARS with presence of hyperinflammation and bacterial superinfection, as well as organ damage and disease severity.

\section{Materials and methods}

\section{Study design}

This study was performed on the Cincinnati Emergency Department (ED) COVID-19 cohort. Adults ( $\geq 18$ years) who presented to the ED of the University of Cincinnati Medical Center (UCMC) between April-May 2020 with COVID-19 symptoms and underwent a clinically-necessary blood draw were preliminarily recruited in this prospective, observational study. Only patients with positive result on standard of care reverse transcriptase polymerase chain reaction (RT-PCR) test for SARS-CoV-2 in nasopharyngeal swab could be included in the final cohort. Patients with negative RT-PCR, or $<18$ years of age at time of presentation, or known prisoners, were excluded. This study was approved by the Institutional Review Board (IRB) of the University of Cincinnati and performed under a waiver of informed consent as it was deemed no more than minimal risk. This study was performed in agreement with the Declaration of Helsinki and in compliance with local and national regulations.

\section{Sample collection and processing}

Blood samples ( $50 \mathrm{~mL}$ as permitted by IRB) were collected at index ED visit during a clinically routine blood draw. Samples were centrifuged at $2,000 \mathrm{~g}$ for $15 \mathrm{~min}$ at $4{ }^{\circ} \mathrm{C}$ and subsequently frozen at $-80{ }^{\circ} \mathrm{C}$ until measurement. All laboratory analyses were performed at the Clinical Nephrology Lab of the Cincinnati Children's Hospital Medical Center, with exception of routine complete blood cell counts (CBC) and serum creatinine, which were performed at the central laboratory of the UCMC. The CBC with differential was performed using a Beckman Coulter UniCel DxH 800 Cellular Analysis System (Brea, CA, USA). Serum creatinine was measured using a kinetic alkaline picrate (modified Jaffe) method on either a Beckman Coulter AU480 Chemistry Analyzer (Brea, California, USA) or a Beckman Coulter AU5822 Chemistry Analyzer (Brea, CA, USA).

\section{Measurement of cytokines and other biomarkers}

Plasma concentrations of interferon (IFN)- $\alpha 2 a$, IFN- $\gamma$, interleukin (IL)-1 $\beta$, IL-6, IL-8, IL-10, IL-1 receptor antagonist (IL-1RA), and tumor necrosis factor- $\alpha$ (TNF- $\alpha$ ) were quantified using Meso Scale Discovery (MSD) U-Plex assay (Rockville, Maryland, USA). Monocyte chemoattractant protein-1 (MCP-1) (R\&D Systems, Minneapolis, MN, USA) and CD163 (Diapharma Group, Inc., West Chester, OH, USA) were assayed using an enzyme linked immunosorbent assays (ELISA). Plasma concentrations of fibrinogen, ferritin, myoglobin, haptoglobin, and C-reactive protein were measured using a BN II System (Siemens Medical Solutions USA, Inc., Malvern, PA, USA). Lactate dehydrogenase was measured on Dimension RxL Max Integrated 
Chemistry System (Siemens Medical Solutions USA, Inc, Malvern, PA, USA), while procalcitonin was tested with a chemiluminescent immunoassay (CLIA) on the Diasorin Liaison XL (DiaSorin S.p.A. Saluggia, Italy). All assays were performed according to manufacturers' instructors and recommendations.

\section{Data collection}

Data on patient demographics, past medical history, presenting vital signs, and clinical course were extracted from electronic medical records (EMR) and recorded into a REDCap (Research Electronic Data Capture) database. An ED physician performed the data extraction, with select records checked by a second ED physician for accuracy. Data on the clinical outcomes of hospitalized patients was recorded until discharge, while information on clinical course of patients discharged at index ED visit were monitored for 30 days.

\section{Outcomes}

The primary cytokines of interest for evaluating the compensatory anti-inflammatory response to COVID-19 were IL-10 and the ratios IL-10/lymphocyte count and IL-10/TNF- $\alpha$, which have been previously used as markers of immunoparalysis $[11,12]$. The primary outcome was maximum severity during hospitalization or within 30 days of index ED presentation. Patient severity was first quantified using a modified version of 8-point ordinal scale as outlined by the World Health Organization (WHO) R\&D Blueprint [13]: (i) not hospitalized/ambulatory, no limitations of activities; (ii) not hospitalized/ambulatory, limitation of activities; (iii) hospitalized, not requiring oxygen therapy; (iv) hospitalized, requiring supplemental oxygen by mask or nasal cannula; (v) hospitalized, on non-invasive ventilation or high flow oxygen devices, or requiring intensive care unit admission; (vi) hospitalized, on invasive mechanical ventilation; (vii) mechanically ventilated and suffering from multi-organ dysfunction syndrome requiring vasopressors or extracorporeal membrane oxygenation (ECMO) or renal replacement therapy; and (viii) death. The highest ordinal scale value was documented. Patients were then classified into one of 3 severity groups, as follows: mild (ordinal scale 1-2), moderate (ordinal scale 3-4) or severe (ordinal scale 5-8). A summary of the COVID-19 severity classification employed in this study is shown in Supplemental Table 1.

The secondary outcomes of this study were COVID-19 severity as described above at ED disposition, and development of severe acute kidney injury (AKI) during hospitalization defined as Kidney Disease Improving Global Outcomes (KDIGO) stages 2 and 3, based on serum creatinine criteria [14]. The tertiary outcome was new positive blood, urine, or other (sputum, bronchoalveolar lavage, etc.) bacterial culture throughout the course of hospitalization.

\section{Statistical analysis}

Categorical data were reported as absolute number (n) and relative frequency (\%), whilst continuous variables were reported as median and interquartile range (IQR). According to expected accounts, categorical variables were compared using the chi-squared test $\left(\chi^{2}\right)$ or Fisher's exact test, as appropriate. Continuous variables were compared using the Mann-Whitney U test. Ratios were calculated to characterize the relationships between pro-inflammatory, anti-inflammatory, and antiviral cytokines. Associations between cytokines vales were tested with Spearman's correlation. Multivariable logistic regression was employed to identify select laboratory variables (IL-6, Il-8, and IL-10) independently predicting primary and secondary outcomes. All baseline patient characteristics from Table 1 with a $p$-value of $<0.10$ were included in the model, and relevant variable selection was performed using the stepwise algorithm. Adjusted odds ratios (ORs) and 95\% confidence intervals (95\% CIs) were calculated. Additional ORs and 95\% CIs were calculated for one standard deviation increases in select laboratory variables (IL-6, Il-8, and IL-10), to allow for those variables to be compared regarding strength of association. As this work represents a pilot, hypothesis-generating study, we did not adjust for multiple comparisons. Statistical analysis was performed using Prism 8 (GraphPad Software, San Diego, CA, USA) and R (version 4.0.2, R Foundation for Statistical Computing, Vienna, Austria) with a $\mathrm{p}<0.05$ considered statistically significant.

\section{Results}

\section{Patient cohort and outcomes}

A total of 52 patients with laboratory-confirmed COVID-19 were enrolled in the cohort, whose essential characteristics are shown in Table 1. At index ED visit, 46 (88.5\%) patients had mild/moderate disease, while 6 (11.5\%) presented with severe disease. Over the course of illness, 10 patients with mild/moderate disease progressed to severe disease, so that a total number of $16(30.8 \%)$ patients reached the primary endpoint of severe COVID-19 within 30 days of index ED visit. Patients with severe disease were a median difference of 20 years older than patients with mild/moderate disease $(p=0.004)$. The only co-morbidity associated with severe disease in the cohort was chronic obstructive pulmonary disease, which was observed in $37.5 \%$ of patients with severe disease as opposed to only $5.5 \%$ with mild disease $(\mathrm{p}=0.007)$. A total of $12(23.1 \%)$ patients reached the secondary endpoint of severe AKI during hospitalization, five patients with stage 2 disease and seven patients with stage 3 disease. Eight (15.4\%) patients reached the tertiary outcome of a new positive bacterial culture during hospitalization, 2 with moderate and 6 with severe COVID-19.

\section{Inflammatory biomarkers and cytokines}

Data on inflammatory biomarkers and cytokines stratified by severity at ED disposition and maximum 30-day severity are presented in Table 2. IL-6, IL-8, and IL-10 were significantly elevated in patients with severe disease both at ED disposition and within 30 days of index ED visit (all $\mathrm{p}<0.01)$. IL-1RA was only elevated in patients with severe 
Table 1: Baseline characteristics of COVID-19 positive patients.

\begin{tabular}{|c|c|c|c|c|}
\hline \multirow[t]{2}{*}{ Variable } & \multirow[t]{2}{*}{ All patients $(n=52)$} & \multicolumn{3}{|c|}{ Maximum 30-day severity } \\
\hline & & Mild/Moderate $(n=36)$ & Severe $(n=16)$ & p-Value \\
\hline Age, years: Median (IQR) & $51(39-66)$ & $46(36-59)$ & $66(53-71)$ & 0.004 \\
\hline Sex (male), n (\%) & $30(57.7 \%)$ & $20(55.5 \%)$ & $10(62.5 \%)$ & 0.639 \\
\hline \multicolumn{5}{|l|}{ Comorbidities, n (\%) } \\
\hline Coronary artery disease & $8(15.4 \%)$ & $5(13.9 \%)$ & $3(18.8 \%)$ & 0.689 \\
\hline Heart failure & $9(17.3 \%)$ & $4(11.1 \%)$ & $5(31.3 \%)$ & 0.113 \\
\hline Hypertension & $26(50.0 \%)$ & $15(41.7 \%)$ & $11(68.8 \%)$ & 0.071 \\
\hline Hyperlipidemia & $15(28.8 \%)$ & $9(25.0 \%)$ & $6(37.5 \%)$ & 0.358 \\
\hline Diabetes & $21(40.4 \%)$ & $16(44.4 \%)$ & $5(31.3 \%)$ & 0.370 \\
\hline Chronic obstructive pulmonary disease & $8(15.4 \%)$ & $2(5.5 \%)$ & $6(37.5 \%)$ & 0.007 \\
\hline Asthma & $8(15.4 \%)$ & $6(16.7 \%)$ & $2(12.5 \%)$ & 1.000 \\
\hline Chronic kidney disease & $6(11.5 \%)$ & $3(8.3 \%)$ & $3(18.8 \%)$ & 0.356 \\
\hline Chronic liver disease & $7(13.5 \%)$ & $3(8.3 \%)$ & $4(25.0 \%)$ & 0.182 \\
\hline Cerebrovascular disease & $7(13.5 \%)$ & $3(8.3 \%)$ & $4(25.0 \%)$ & 0.182 \\
\hline Cancer & $4(8.0 \%)$ & $1(2.8 \%)$ & $3(18.8 \%)$ & 0.081 \\
\hline Obesity & $16(30.8 \%)$ & $11(30.6 \%)$ & $5(31.3 \%)$ & 0.960 \\
\hline Inherited immunodeficiency & $0(0 \%)$ & $0(0 \%)$ & $0(0 \%)$ & - \\
\hline Acquired immunodeficiency (HIV, transplant) & $3(5.8 \%)$ & $1(2.8 \%)$ & $2(12.5 \%)$ & 0.165 \\
\hline Autoimmune disease & $2(3.8 \%)$ & $1(2.8 \%)$ & $1(6.3 \%)$ & 0.547 \\
\hline Current smoker & $12(23.1 \%)$ & $7(19.4 \%)$ & $5(31.3 \%)$ & 0.351 \\
\hline Former smoker & $11(21.2 \%)$ & $7(19.4 \%)$ & $4(25.0 \%)$ & 0.650 \\
\hline \multicolumn{5}{|l|}{ Symptoms at presentation, $\mathbf{n}(\%)$} \\
\hline Fever & $27(51.9 \%)$ & $18(50.0 \%)$ & $9(56.3 \%)$ & 0.677 \\
\hline Fatigue & $9(17.3 \%)$ & $4(11.1 \%)$ & $5(31.3 \%)$ & 0.113 \\
\hline Abdominal pain & $4(8.0 \%)$ & $3(8.3 \%)$ & $1(6.3 \%)$ & 1.000 \\
\hline Nausea & $9(17.3 \%)$ & $8(22.2 \%)$ & $1(6.3 \%)$ & 0.245 \\
\hline Vomiting & $3(5.8 \%)$ & $2(5.6 \%)$ & $1(6.3 \%)$ & 1.000 \\
\hline Diarrhea & 7 (13.5\%) & $2(5.6 \%)$ & $5(31.3 \%)$ & 0.023 \\
\hline Headache & $6(11.5 \%)$ & $6(16.7 \%)$ & $0(0 \%)$ & 0.160 \\
\hline Sore throat & $4(8.0 \%)$ & $4(11.1 \%)$ & $0(0 \%)$ & 0.299 \\
\hline Loss of smell & $2(3.8 \%)$ & $1(2.8 \%)$ & $0(0 \%)$ & 1.000 \\
\hline Loss of taste & $2(3.8 \%)$ & $1(2.8 \%)$ & $1(6.3 \%)$ & 0.525 \\
\hline Cough & $24(46.2 \%)$ & $17(47.2 \%)$ & $7(43.8 \%)$ & 1.000 \\
\hline Dyspnea & $32(61.5 \%)$ & $23(63.9 \%)$ & $9(56.3 \%)$ & 0.759 \\
\hline Chest pain & $12(23.1 \%)$ & $8(22.2 \%)$ & $4(25.0 \%)$ & 1.000 \\
\hline Myalgia & $8(15.4 \%)$ & $8(22.2 \%)$ & $0(0 \%)$ & 0.047 \\
\hline Days since symptom onset: Median (IQR) & $7(3-10)$ & $7(4-9)$ & $5(1-10)$ & 0.551 \\
\hline
\end{tabular}

disease at ED disposition ( $p=0.048)$, while TNF- $\alpha$ was marginally elevated in those who developed severe disease within 30 days of their index ED visit $(p=0.049)$. To compare the relationship between pro- and antiinflammatory responses in COVID-19, we correlated IL-6 with IL-10, and a significant positive correlation was observed $(r=0.748 ; p<0.001)$.

Data on cytokine ratios evaluating CARS (immune dysfunction) by severity at ED disposition and maximum 30-day severity are presented in Table 3. Two markers of CARS, IL-10/TNF- $\alpha$ and IL-10/lymphocyte count, were significantly elevated in patients with severe disease at both time points (all $\mathrm{p}<0.05$ ). The IL-6/lymphocyte count ratio was significantly elevated in patients with severe disease at ED disposition $(\mathrm{p}=0.014)$ and within 30-days $(\mathrm{p}<0.001)$.

Data on IL-10, IL-10/lymphocyte count, and IL-10/ TNF- $\alpha$ with respect to secondary and tertiary outcome is presented in Table 4. IL-10 and IL-10/lymphocyte count were significantly elevated in patients who developed severe AKI during hospitalization and in those with who developed a new positive bacterial culture (all p's $\leq 0.01$ ). IL-10/TNF- $\alpha$ ratio was not associated with either outcome.

IL-10 and IL-10/lymphocyte count ratio were analyzed in multivariate logistic regression for independent associated with outcomes (Table 5). After adjusting for 
Table 2: Inflammatory and cytokine profile of mild/moderate vs. severe COVID-19 patients.

\begin{tabular}{|c|c|c|c|c|c|c|}
\hline \multirow[t]{2}{*}{ Lab variable } & \multicolumn{3}{|c|}{ ED disposition severity } & \multicolumn{3}{|c|}{ Maximum 30-day severity } \\
\hline & $\begin{array}{r}\text { Mild/Moderate } \\
(n=46)\end{array}$ & Severe $(n=6)$ & p-Value & $\begin{array}{r}\text { Mild/Moderate } \\
(n=36)\end{array}$ & Severe $(n=16)$ & p-Value \\
\hline White blood cell count, $\times 10^{9} / \mathrm{L}$ & $6.3(5.3-9.3)$ & $9.6(4.8-15.9)$ & 0.319 & $6.7(5.2-9.3)$ & $6.8(5.3-9.6)$ & 0.862 \\
\hline Neutrophil count, $\times 10^{9} / \mathrm{L}$ & $4.4(3.5-6.7)$ & $8.2(3.4-12.9)$ & 0.151 & $5.2(3.9-8.8)$ & $4.4(3.3-6.7)$ & 0.212 \\
\hline Lymphocyte count, $\times 10^{9} / \mathrm{L}$ & $1.0(0.7-1.4)$ & $0.9(0.4-1.5)$ & 0.483 & $1.0(0.8-1.6)$ & $0.8(0.6-1.2)$ & 0.143 \\
\hline Platelet count, $\times 10^{9} / \mathrm{L}$ & $206(164-263)$ & $220(134-270)$ & 0.994 & $203.5(163.3-252.0)$ & $211.5(152.5-346.8)$ & 0.484 \\
\hline Neutrophil-to-lymphocyte ratio & $5.3(2.8-7.0)$ & $8.7(5.6-16.8)$ & 0.056 & $5.3(2.7-6.7)$ & $6.8(4.3-9.7)$ & 0.176 \\
\hline \multirow[t]{2}{*}{ Platelet-to-lymphocyte ratio } & 183.7 & 235.8 & 0.523 & 181.2 & 256.4 & 0.048 \\
\hline & $(134.2-296.4)$ & (154.4-485.9) & & $(118.2-267.5)$ & $(169.8-415.1)$ & \\
\hline C-reactive protein, $\mathrm{mg} / \mathrm{L}$ & $48.0(8.0-121.3)$ & $75.0(16.0-139.0)$ & 0.456 & $48.0(7.0-122.0)$ & $49.0(27.0-123.0)$ & 0.223 \\
\hline Procalcitonin, $\mu \mathrm{g} / \mathrm{L}$ & $0.08(0.05-0.28)$ & $0.32(0.15-3.03)$ & 0.044 & $0.07(0.05-0.20)$ & $0.26(0.11-0.77)$ & 0.026 \\
\hline \multirow[t]{2}{*}{ Ferritin, $\mu \mathrm{g} / \mathrm{L}$} & 331.5 & 584.5 & 0.251 & 251.5 & 1032.0 & 0.019 \\
\hline & $(112.8-1288.0)$ & $(344.3-1943.0)$ & & $(106.0-1018.0)$ & $(232.5-1475.0)$ & \\
\hline \multirow[t]{2}{*}{ Lactate dehydrogenase, $\mathrm{U} / \mathrm{L}$} & 307.5 & 331.5 & 0.809 & 280.0 & 407.0 & 0.003 \\
\hline & $(243.8-453.0)$ & $(254.8-506.0)$ & & $(225.8-382.5)$ & $(311.0-576.0)$ & \\
\hline \multirow[t]{2}{*}{ Haptoglobin, mg/L } & 2540.0 & 2535.0 & 0.454 & 2445.0 & 2690.0 & 0.378 \\
\hline & $(1585.0-3323.0)$ & $(2195.0-4313.0)$ & & $(1575.0-3315.0)$ & $(2155.0-3778.0)$ & \\
\hline Myoglobin, $\mu \mathrm{g} / \mathrm{L}$ & $28.4(17.4-96.8)$ & $242.5(83.5-367.0)$ & 0.047 & $24.9(15.3-65.2)$ & $108.0(34.9-342.9)$ & 0.009 \\
\hline IFN- $\alpha 2 a, n g / L$ & $13.1(3.4-30.6)$ & $18.9(4.9-28.6)$ & 0.837 & $13.3(3.7-25.0)$ & $15.6(4.6-40.7)$ & 0.797 \\
\hline IFN-,$- n g / L$ & $73.6(40.9-279.5)$ & $158.1(20.2-655.0)$ & 0.738 & $67.7(30.0-276.9)$ & $193.5(40.9-806.6)$ & 0.260 \\
\hline $\mathrm{IL}-1 \beta, \mathrm{ng} / \mathrm{L}$ & $0.18(0.06-0.42)$ & $0.78(0.16-2.1)$ & 0.138 & $0.16(0.05-0.35)$ & $0.36(0.16-0.77)$ & 0.071 \\
\hline IL-6, ng/L & $14.7(4.9-33.3)$ & $63.2(30.1-153.6)$ & 0.004 & $9.7(2.3-19.0)$ & $36.4(16.3-49.4)$ & $<0.001$ \\
\hline IL-8, ng/L & $12.6(7.9-23.5)$ & $73.8(16.0-93.0)$ & 0.005 & $11.4(5.4-17.4)$ & $26.8(17.4-56.6)$ & $<0.001$ \\
\hline IL-10, ng/L & $1.2(0.5-2.5)$ & $5.9(5.0-29.8)$ & $<0.001$ & $0.9(0.3-2.2)$ & $5.3(1.8-6.4)$ & $<0.001$ \\
\hline \multirow[t]{2}{*}{ IL-1RA, ng/L } & 1024.0 & 2140.0 & 0.049 & 1024.0 & 2055.0 & 0.083 \\
\hline & $(443.6-2530.0)$ & $(1518.0-3860.0)$ & & $(421.5-1908.0)$ & $(842.2-3012.0)$ & \\
\hline TNF- $\alpha, n g / L$ & $2.7(2.0-5.1)$ & $8.8(2.5-12.1)$ & 0.121 & $2.6(2.1-4.0)$ & $5.2(2.1-11.9)$ & 0.049 \\
\hline \multirow[t]{2}{*}{ CD163, $\mu \mathrm{g} / \mathrm{L}$} & 1075.0 & 845.5 & 0.685 & 1055.0 & 1130.0 & 0.473 \\
\hline & $(3.2-1701.0)$ & $(5.3-1707.0)$ & & $(2.9-1555.0)$ & $(118.2-2567.0)$ & \\
\hline \multirow[t]{2}{*}{ MCP-1, ng/L } & 530.6 & 778.6 & 0.121 & 501.6 & 713.1 & 0.057 \\
\hline & $(384.8-795.9)$ & (556.2-1727.0) & & $(357.4-752.8)$ & (467.4-1009.0) & \\
\hline
\end{tabular}

All data presented as median (IQR). IL, interleukin; TNF, tumor necrosis factor.

Table 3: Cytokine and inflammatory ratios.

\begin{tabular}{|c|c|c|c|c|c|c|}
\hline \multirow[t]{2}{*}{ Ratio } & \multirow[b]{2}{*}{ Mild/Moderate $(n=46)$} & \multicolumn{2}{|c|}{ ED disposition severity } & \multicolumn{3}{|c|}{ Maximum 30-day severity } \\
\hline & & Severe $(n=6)$ & p-Value & Mild/Moderate $(n=36)$ & Severe $(n=16)$ & p-Value \\
\hline IL-6/IL-10 & $7.0(5.4-13.7)$ & $8.5(2.1-27.1)$ & 0.827 & $7.4(5.5-13.4)$ & $6.9(4.6-19.8)$ & 0.734 \\
\hline IL-10/TNF- $\alpha$ & $0.32(0.15-0.88)$ & $1.3(0.48-9.5)$ & 0.021 & $0.3(0.3-0.8)$ & $0.6(0.5-3.3)$ & 0.020 \\
\hline IFN-ץ/IL-10 & $83.4(42.4-157.9)$ & $25.1(2.2-25.1)$ & 0.07 & $92.5(49.2-141.7)$ & $43.2(19.9-148.7)$ & 0.094 \\
\hline IL-6/lymphocyte count & $12.7(4.8-39.8)$ & $51.7(23.7-415.9)$ & 0.014 & $11.5(3.9-27.0)$ & $39.5(16.5-81.4)$ & 0.001 \\
\hline IL-10/lymphocyte count & $1.6(0.50-3.1)$ & $11.4(7.9-31.0)$ & 0.008 & $1.1(0.4-2.6)$ & $6.4(1.6-11.6)$ & $<0.001$ \\
\hline
\end{tabular}

All data presented as median (IQR). IL, interleukin; TNF, tumor necrosis factor.

Table 4: Compensatory anti-inflammatory response, acute kidney injury, and new positive bacterial cultures infections.

\begin{tabular}{|c|c|c|c|c|c|c|}
\hline Variable & $\begin{array}{r}\text { No severe AKI } \\
\text { (None or KDIGO 1) }\end{array}$ & $\begin{array}{r}\text { Severe AKI } \\
\text { (KIDGO 2 + 3) }\end{array}$ & p-Value & $\begin{array}{r}\text { No positive bacterial } \\
\text { cultures }\end{array}$ & $\begin{array}{r}\text { New positive bacterial } \\
\text { culture }\end{array}$ & p-Value \\
\hline IL-10 & $1.2(0.5-2.5)$ & $5.6(1.5-8.5)$ & 0.007 & $1.2(0.5-2.5)$ & $5.8(2.6-8.1)$ & 0.001 \\
\hline IL-10/TNF- $\alpha$ & $0.4(0.2-0.9)$ & $0.5(0.1-3.7)$ & 0.761 & $0.3(0.2-0.9)$ & $0.5(0.3-3.3)$ & 0.204 \\
\hline IL-10/lymphocyte count & $1.6(0.5-3.0)$ & $8.4(1.1-11.8)$ & 0.010 & $1.6(0.5-3.0)$ & $9.2(3.4-11.6)$ & 0.003 \\
\hline
\end{tabular}

All data presented as median (IQR). IL, interleukin; TNF, tumor necrosis factor; AKI, acute kidney injury. 
Table 5: Results of multivariate logistic regressions for severe COVID-19 and severe acute kidney injury.

\begin{tabular}{|c|c|c|c|c|c|}
\hline Outcome & Covariables & Estimate & Standard error & p-Value & OR $(95 \% \mathrm{Cl})$ \\
\hline \multirow[t]{2}{*}{ Maximum 30-day COVID-19 severity } & Age & 0.076 & 0.031 & 0.013 & $1.08(1.02-1.15)$ \\
\hline & IL-10 & 0.354 & 0.164 & 0.031 & $1.42(1.03-1.96)$ \\
\hline \multirow[t]{2}{*}{ Severe AKI } & Number of comorbidities & 0.336 & 0.141 & 0.017 & $1.39(1.06-1.84)$ \\
\hline & IL-10 & 0.337 & 0.160 & 0.035 & $1.40(1.02-1.92)$ \\
\hline \multirow[t]{2}{*}{ Maximum 30-day COVID-19 severity } & Age & 0.074 & 0.032 & 0.004 & $1.08(1.01-1.15)$ \\
\hline & IL-10/Lymphocyte count & 0.279 & 0.113 & 0.013 & $1.32(1.06-1.65)$ \\
\hline \multirow[t]{2}{*}{ Severe AKI } & Age & 0.031 & 2058 & 0.039 & $1.07(1.01-1.14)$ \\
\hline & IL-8 & 0.019 & 2613 & 0.009 & $1.05(1.01-1.09)$ \\
\hline \multirow[t]{2}{*}{ Maximum 30-day COVID-19 severity } & Age & 0.082 & 0.030 & 0.006 & $1.09(1.02-1.15)$ \\
\hline & IL-8 & 0.059 & 0.023 & 0.010 & $1.06(1.01-0.11)$ \\
\hline \multirow[t]{2}{*}{ Maximum 30-day COVID-19 severity } & Age & 0.071 & 0.029 & 0.014 & $1.07(1.02-1.137)$ \\
\hline & IL-6 & 0.042 & 0.019 & 0.027 & $1.044(1.01-1.08)$ \\
\hline
\end{tabular}

confounders, a one-unit increase in IL-10 was associated $42 \%$ increased odds of severe COVID-19 within 30 days of index ED visit ( $\mathrm{p}=0.031$ ), whilst a one-unit increase IL-10/ lymphocyte count ratio was also associated with $32 \%$ increased in odds of odds of severe COVID-19 within 30 days of index ED visit ( $\mathrm{p}=0.013)$. Only IL-10 was found to be predictive of severe AKI in multivariate analysis, with a one-unit increase associated with $40 \%$ increased odds of this outcome. Logistic regression for new positive bacterial cultures was prohibited due to the low number of patients experiencing this complication.

IL-6 and IL-8 counts were also analyzed in separate multivariate logistic regressions, to prevent collinearity issues. The results are presented in Table 5. Both IL-6 and IL-8 were found to be significantly associated with severe disease when adjusted for demographic confounders, with a one-unit increase being responsible for a 4 and $6 \%$ increase in odds of progression to severe disease, respectively. IL-8 was also associated with severe AKI, while IL-6 was not. Since IL-10, IL-8 and IL-6 raw values naturally vary in different intervals and cannot be considered directly comparable, ORs for progression to severe disease and severe AKI were calculated considering a difference of one standard deviation for each parameter, and are available in Table 6. An IL-10 increase was found to be substantially more strongly associated with both progression to severe disease and severe AKI.

\section{Discussion}

The results of this study demonstrate that the proinflammatory response to SARS-CoV-2 infection is accompanied by a substantial anti-inflammatory response, which is associated with disease severity even when measured at
ED presentation. While the hyperinflammatory consequences of COVID-19 has been the focus of much debate, our study emphasizes the association between the antiinflammatory cytokine response and adverse outcomes in COVID-19. Hence, the notion of COVID-19 as a purely hyperinflammatory condition is likely to be a flawed oversimplification. Indeed, recent studies have failed to observe a cytokine storm phenomenon in critically COVID-19 patients $[4,5]$. The relative contribution of hyperinflammation and immunoparalysis would be expected to differ among patients and during the different stages of disease, which complicates treatment strategies and clinical trials using immunomodulatory strategies. Immunomodulation must be tailored to the underlying immunobiology for any given patient, and we support the proposition of Hall et al. that personalized immunophenotype-based immunotherapy is likely to yield superior outcomes [15]. Failure of IL-6 inhibition in clinical trials may have resulted from a heterogeneous group, including patients with significant immunoparalysis and variable immunophenotypes at time of administration.

In this investigation, we observed an independent association between disease severity and IL-10 and IL-10/ Lymphocyte Count Ratio. In sepsis, high values of IL-10 are

Table 6: Adjusted odds ratios for increase of one standard deviation in interleukin (IL)-6, 8, and 10.

\begin{tabular}{llrr}
\hline Outcome & Parameter & $\begin{array}{l}\text { Standard } \\
\text { deviation }\end{array}$ \\
\hline Maximum 30-day & IL-10 & 10.97 & $48.19(34.95-66.44)$ \\
COVID-19 severity & IL-8 & 22.41 & $3.75(3.59-3.93)$ \\
& IL-6 & 42.41 & $5.94(5.72-6.16)$ \\
Severe AKI & IL-10 & 10.97 & $40.13(29.34-54.88)$ \\
& IL-8 & 22.41 & $1.53(0.01-256.54)$ \\
\hline
\end{tabular}


associated with poor outcomes, including higher mortality [16]. In a finding similar to COVID-19, the CARS of severe sepsis is associated with high IL-10 and lymphocyte depletion [12]. Li et al. reported that the IL-10/Lymphocyte Count Ratio was associated with severity and outcome of infection in sepsis patients admitted to the intensive care unit [12]. In COVID-19, the high IL-10 appears to be driven by the overall dysregulated inflammatory response as denoted by the significant correlation with IL-6 observed in this study. However, this immune dysregulation likely inhibits the utility of directly correlating pro- vs. anti-inflammatory cytokines. As found in this study, though IL-10/TNF- $\alpha$ ratio was significant when comparing levels by disease severity in univariate analysis, this was not confirmed in multivariate analysis. As such, comparing a cytokine with a cellular marker may yield more informative assessment. Nonetheless, as observed in the multivariate analysis, though IL-6 and IL-8 were associated with progression to severe disease, when standardized to allow for direct comparison, IL-10 was substantially more strongly associated with both disease progression and development of severe AKI then the proinflammatory cytokines. In addition to a dysregulated immune response driving cytokine production, preliminary evidence from Davanzo et al. demonstrated that SARS-CoV-2 infects lymphocytes directly via CD4, and that such infected cells produce high amounts of IL-10, which was associated with viral persistence and disease severity [17].

It has been clearly established that low lymphocyte count is associated with poor disease progression and outcomes in COVID-19 $[18,19,20]$. The mechanism for the observed low lymphocyte counts in patients with severe COVID-19 is not fully elucidated, but likely involves a combination of disrupted bone marrow generation, cell depletion, apoptosis, and direct viral cytopathic insults [21]. Compounding these, IL-10 may play a part in the apoptosis of lymphocytes, as observed in sepsis $[22,23,24]$. Yet both T-cell and B-cell lymphocytes secrete IL-10 [25], thus demonstrating the intricate relationship between these two measures of immune status. The combination of lymphopenia with elevated IL-10 potentially reflects a state of functional immunoparalysis, which would be expected to impair both innate and adaptive antimicrobial responses.

Along with its association with disease severity, elevated IL-10 levels at ED presentation were also found to be independently associated with increased odds of developing severe AKI, while IL-6 was not. At first glance, this may be relatively surprising, as IL-10 is a potent antiinflammatory cytokine that inhibits the inflammatory and cytotoxic pathways that perpetuate AKI [26]. Animal models have demonstrated that IL-10 attenuates inflammation and AKI due to a variety of insults, including ischemic injury [27]. This is thought to occur for a number of mechanisms, including the inhibition of genes triggering leukocyte activation and adhesion, and inducing inducible nitric oxide synthase [27]. However, Zhang et al. reported that IL-10 elevation was associated with AKI (adjusted OR: 1.57 [95\% CI, 1.04 to 2.38]) but lower risk of mortality (adjusted hazard ratio, 0.72 [95\% CI, 0.56 to 0.93$]$ ) in adults after cardiac surgery [28]. In part, IL-10 exerts its protective renal effects through induction of neutrophil gelatinaseassociated lipocalin (NGAL) [29], which itself is an early and sensitive diagnostic and prognostic serum and urine biomarker of AKI, which can rise $24 \mathrm{~h}$ earlier than creatinine [30]. In further analysis, Zhang et al. observed that improved outcomes in patients with high IL-10 levels could only be seen in those with concomitant elevations in NGAL, while those with high IL-10 and low NGAL were afforded a worse prognosis [28]. Such findings suggest that IL-10 may only be protective when it induces NGAL expression [28]. NGAL should be measured and correlated with IL-10 in future studies on patients with COVID-19 to assess this relationship, as well as for assessing overt AKI and subclinical renal injury in patients with COVID-19.

Unsurprisingly, IL-10 and IL-10/lymphocyte count were significantly elevated in patients who developed new positive bacterial cultures during their COVID-19 course. This finding suggests a direct clinical consequence of a antiinflammatory cytokine response in some patients with severe COVID-19 driven by high IL-10 blunting a proinflammatory antimicrobial response with low lymphocyte count impairing adaptive immunity. In general, secondary infections are associated with increased morbidity and mortality in viral respiratory infections, including COVID-19 $[31,32,33]$. In a comprehensive meta-analysis, Langford et al. reported a prevalence rate of $14.3 \%$ (95\%CI: 9.6-18.9\%) for secondary bacterial infections in patients with COVID-19 [34]. However, the authors argued against empiric antibacterial therapy [34]. Elevated IL-10/lymphocyte count ratio has the potential to inform empiric antibiotic decisionmaking and should be the subject of prospective study. Due to the limited number of subjects with new positive bacterial cultures in our study, we could not evaluate this variable in multivariate analysis. However, this should be further investigated in future studies.

This study was limited by a relatively small sample size and a single time point of laboratory measurements. We choose ED presentation as a time point of clinical significance, as it is not only the point when a patient felt sick enough to seek medical care, but it often presents one of the earliest and perhaps most efficient opportunity for intervention. Although patients' immunologic state may significantly vary over the course of illness, we were able to capture 
patients at a variety of stages at index ED visit, including severe disease. Nonetheless, future studies should be performed to serially monitor biomarkers of immunoparalysis throughout hospitalization. Moreover, future molecular studies are needed to characterize the CARS response, including HLA-DR monocyte expression and ex vivo LPS-induced TNF- $\alpha$ release. Finally, when pre-COVID-19 serum creatinine value was not available, the index ED visit serum creatinine was used in such cases as a baseline value for evaluating AKI. Thus, it is possible that cases of AKI were missed in patients who presented with peak creatinine value resolving during hospitalization. As such, the incidence of this endpoint may have been underrepresented.

\section{Conclusions}

The hyperinflammatory response to COVID-19 is accompanied by a simultaneous CARS that is associated with poor outcomes and may increase the new positive bacterial cultures. IL-10 and IL-10/lymphocyte count ratio at ED presentation were independent predictors of COVID-19 severity, while IL-10 levels predicted development of AKI. Interestingly, the anti-inflammatory cytokine IL-10 is more strongly associated with disease progression and severe AKI than the proinflammatory cytokines IL- 6 and IL-8. The CARS phenomenon further introduces heterogeneity into COVID-19 population, and may mask effects of potentially useful antiinflammatory drugs for patients without substantial CARS in clinical trials. The CARS during COVID-19 requires further investigation to enable more precise immunomodulatory therapy against SARS-CoV-2, which should be optimally tailored to an individual patient's immunophenotype.

Research funding: This study was funded by the University of Cincinnati College of Medicine Special Coronavirus (COVID-19) Research Pilot Grant Program. James Rose is funded by the NIH Pediatric Center of Excellence in Nephrology P50DK096418 (PI: Devarajan, Prasad).

Author contributions: All authors have accepted responsibility for the entire content of this manuscript and approved its submission.

Competing interests: Authors state no conflict of interest. Informed consent: Informed consent was obtained from all individuals included in this study.

Ethical approval: This study was approved by the Institutional Review Board (IRB) of the University of Cincinnati and performed under a waiver of informed consent as it was deemed no more than minimal risk. This study was performed in agreement with the Declaration of Helsinki and in compliance with local and national regulations.

\section{References}

1. Ragab D, Salah Eldin H, Taeimah M, Khattab R, Salem R. The COVID-19 cytokine storm; what we know so far. Front Immunol 2020;11:1446.

2. Zhang C, Wu Z, Li J-W, Zhao H, Wang G-Q. Cytokine release syndrome in severe COVID-19: interleukin-6 receptor antagonist tocilizumab may be the key to reduce mortality. Int J Antimicrob Agents 2020;55:105954.

3. Roche. Roche provides an update on the phase III COVACTA trial of Actemra/RoActemra in hospitalised patients with severe COVID-19 associated pneumonia. [Internet]; 2020. Available from: https://www.roche.com/investors/updates/inv-update2020-07-29.htm [cited 2020 Aug 16].

4. Sinha P, Calfee CS, Cherian S, Brealey D, Cutler S, King C, et al. Prevalence of phenotypes of acute respiratory distress syndrome in critically ill patients with COVID-19: a prospective observational study [Internet]. Lancet Respir Med; 2020 Aug 27. Available from: https://www.thelancet.com/journals/lanres/ article/PIIS2213-2600(20)30366-0/abstract [cited 2020 Oct 14]; $0(0)$.

5. Kox M, Waalders NJB, Kooistra EJ, Gerretsen J, Pickkers P. Cytokine Levels in critically ill patients with COVID-19 and other conditions [Internet]. JAMA; 2020 Sep 3. Available from: https:// jamanetwork.com/journals/jama/fullarticle/2770484 [cited 2020 Oct 14].

6. Hall MW, Greathouse KC, Thakkar RK, Sribnick EA, Muszynski JA. Immunoparalysis in pediatric critical care. Pediatr Clin 2017;64: 1089-102.

7. Jensen IJ, Sjaastad FV, Griffith TS, Badovinac VP. Sepsis-induced T cell immunoparalysis: the ins and outs of impaired T cell immunity. J Immunol 2018 01;200:1543-53.

8. Greathouse KC, Hall MW. Critical illness-induced immune suppression: current state of the science. Am J Crit Care 2016 Jan; 25:85-92.

9. Hall MW, Knatz NL, Vetterly C, Tomarello S, Wewers MD, Volk HD, et al. Immunoparalysis and nosocomial infection in children with multiple organ dysfunction syndrome. Intensive Care Med 2011 Mar;37:525-32.

10. Schulte-Schrepping J, Reusch N, Paclik D, Baßler K, Schlickeiser S, Zhang B, et al. Severe COVID-19 is marked by a dysregulated myeloid cell compartment. Cell 2020 Aug 5;182:1419-1440.e23.

11. Allen ML, Hoschtitzky JA, Peters MJ, Elliott M, Goldman A, James I, et al. Interleukin-10 and its role in clinical immunoparalysis following pediatric cardiac surgery. Crit Care Med 2006 Oct;34: 2658-65.

12. Li X, Xu Z, Pang X, Huang Y, Yang B, Yang Y, et al. Interleukin-10/ lymphocyte ratio predicts mortality in severe septic patients [Internet]. PloS One; 2017 Jun 19. Available from: https://www. ncbi.nlm.nih.gov/pmc/articles/PMC5476240/ [cited 2020 Aug 16];12(6).

13. World Health Organization. WHO R\&D blueprint novel coronavirus: COVID-19 therapeutic trial synopsis [Internet]; 2020. Available from: https://www.who.int/blueprint/ priority-diseases/key-action/COVID-19_Treatment_Trial_ Design_Master_Protocol_synopsis_Final_18022020.pdf [cited 2020 Jun 1].

14. KDIGO AKI Working Group. KDIGO clinical practice guideline for acute kidney injury. Kidney Int Suppl 2012;2:1. 
15. Hall MW, Joshi I, Leal L, Ooi EE. Immune modulation in COVID-19: strategic considerations for personalized therapeutic intervention [Internet]. Clin Infect Dis; 2020. Available from: https://academic.oup.com/cid/advance-article/doi/10.1093/ cid/ciaa904/5865455 [cited 2020 Aug 16].

16. Gogos CA, Drosou E, Bassaris HP, Skoutelis A. Pro-versus antiinflammatory cytokine profile in patients with severe sepsis: a marker for prognosis and future therapeutic options. J Infect Dis 2000 Jan;181:176-80.

17. Davanzo GG, Codo AC, Brunetti NS, Boldrini Vo, Knittel TL, Monterio LB, et al. SARS-CoV-2 Uses CD4 to infect Thelper lymphocytes. medRxiv 2020 Sep 28;2020.09.25.20200329.

18. Henry BM. COVID-19, ECMO, and lymphopenia: a word of caution. Lancet Respir Med 2020 Mar 13;8:e24.

19. Henry B, Cheruiyot I, Vikse J, Mutua V, Kipkorir V, Benoit J, et al. Lymphopenia and neutrophilia at admission predicts severity and mortality in patients with COVID-19: a meta-analysis. 2020 Jul 16;91. 1 [ahead of print-ahead of print].

20. Henry BM, de Oliveira MHS, Benoit S, Plebani M, Lippi G. Hematologic, biochemical and immune biomarker abnormalities associated with severe illness and mortality in coronavirus disease 2019 (COVID-19): a meta-analysis. Clin Chem Lab Med 2020;58:1021-8.

21. Henry BM, Vikse J, Benoit S, Favaloro EJ, Lippi G. Hyperinflammation and derangement of renin-angiotensinaldosterone system in COVID-19: a novel hypothesis for clinically suspected hypercoagulopathy and microvascular immunothrombosis. Clin Chim Acta 2020 Aug 1;507:167-73.

22. Oberholzer A, Oberholzer C, Moldawer LL. Interleukin-10: a complex role in the pathogenesis of sepsis syndromes and its potential as an anti-inflammatory drug. Crit Care Med 2002 Jan; 30(1 Suppl):S58-63.

23. de Waal Malefyt R, Yssel H, de Vries JE. Direct effects of IL-10 on subsets of human CD4+ T cell clones and resting T cells. Specific inhibition of IL-2 production and proliferation. J Immunol 1993 Jun 1;150:4754-65.

24. Taga K, Tosato G. IL-10 inhibits human T cell proliferation and IL-2 production. J Immunol 1992 Feb 15;148:1143-8.

25. Scumpia PO, Moldawer LL. Biology of interleukin-10 and its regulatory roles in sepsis syndromes. Crit Care Med 2005 Dec; 33(12 Suppl):S468-471.
26. Akcay A, Nguyen Q, Edelstein CL. Mediators of inflammation in acute kidney injury. Mediat Inflamm 2009;2009:137072.

27. Deng J, Kohda Y, Chiao H, Wang Y, Hu X, Hewitt SM, et al. Interleukin-10 inhibits ischemic and cisplatin-induced acute renal injury. Kidney Int 2001 Dec;60:2118-28.

28. Zhang WR, Garg AX, Coca SG, Devereaux PJ, Eikelboom J, Kavsak P, et al. Plasma IL- 6 and IL-10 concentrations predict AKI and longterm mortality in adults after cardiac surgery. J Am Soc Nephrol 2015 Dec;26:3123-32.

29. Jung M, Sola A, Hughes J, Kluth DC, Vinuesa E, Viñas JL, et al. Infusion of IL-10-expressing cells protects against renal ischemia through induction of lipocalin-2. Kidney Int 2012 May; 81:969-82.

30. Helanova K, Spinar J, Parenica J. Diagnostic and prognostic utility of neutrophil gelatinase-associated lipocalin (NGAL) in patients with cardiovascular diseases-review. Kidney Blood Press Res 2014;39:623-9.

31. Klein EY, Monteforte B, Gupta A, Jiang W, May L, Hsieh Y-H, et al. The frequency of influenza and bacterial coinfection: a systematic review and meta-analysis. Influenza Other Respir Viruses 2016; 10:394-403.

32. Rice TW, Rubinson L, Uyeki TM, Vaughn FL, John BB, Miller RR, et al. Critical illness from 2009 pandemic influenza A virus and bacterial coinfection in the United States. Crit Care Med 2012 May;40:1487-98.

33. Neto AGM, Lo KB, Wattoo A, Salacup G, Pelayo J, Dejoy R, et al. Bacterial infections and patterns of antibiotic use in patients with COVID-19 [Internet]. J Med Virol; 2020. Available from: https:// onlinelibrary.wiley.com/doi/abs/10.1002/jmv.26441 [cited 2020 Aug 19];n/a(n/a).

34. Langford BJ, So M, Raybardhan S, Leung V, Westwood D, MacFadden DR, et al. Bacterial co-infection and secondary infection in patients with COVID-19: a living rapid review and meta-analysis [Internet]. Clin Microbiol Infecti; 2020 Jul 22. Available from: https://www.clinicalmicrobiologyandinfection. com/article/S1198-743X(20)30423-7/abstract [cited 2020 Aug $16] ; 0(0)$.

Supplementary Material: The online version of this article offers supplementary material (https://doi.org/10.1515/cclm-2020-1284). 